\title{
Variabilidade espacial da resistência do solo à penetração e da taxa de estratificação de carbono orgânico do solo em um Latossolo Vermelho eutroférrico
}

\author{
Spatial variability of soil resistance to penetration and soil organic carbon stratification ratio on a \\ Typic Eutrothox
}

\author{
Jonez Fidalski ${ }^{1}$ Cássio Antonio Tormena² Antonio Carlos Andrade Gonçalves ${ }^{2}$ \\ Rubem Silvério Oliveira Júnior ${ }^{2}$
}

\section{RESUMO}

A compactação e a qualidade do solo são constantemente questionadas para a definição do manejo de solo em plantio direto. Os objetivos deste estudo foram avaliar a variabilidade espacial da resistência do solo à penetração, da densidade do solo e a taxa de estratificação de carbono orgânico do solo em camadas de um Latossolo Vermelho eutroférrico, textura muito argilosa, em áreas contíguas de plantio direto com rotação de culturas e plantio direto com sucessão de culturas associado à escarificação. $O$ estudo foi realizado de maio a junho de 2002, no município de Palotina, região Oeste do Paraná. As amostras para determinação da densidade do solo, da resistência do solo à penetração e do teor de carbono orgânico do solo foram obtidas em 120 pontos, em uma grade de $2 \times 5 \mathrm{~m}$, na profundidade de 0,40m. Foram feitas três medidas de resistência do solo à penetração, em um período de 10 dias, iniciado depois de cessada a precipitação. A densidade do solo não explicou a dependência espacial da compactação do solo. A resistência do solo à penetração e a taxa de estratificação de carbono orgânico do solo apresentam alcances de $17 \mathrm{~m}$. A variação temporal da dependência espacial da resistência do solo à penetração ocorre nas camadas de 0,05-0,20m. As semelhanças da dependência espacial da resistência do solo à penetração e da taxa de estratificação de carbono orgânico do solo, revelaram a importância da qualidade do solo na estabilização espacial e temporal da compactação do solo, expressa pela resistência do solo à penetração em sistemas de plantio direto.

Palavras-chave: geoestatística, plantio direto, densidade do solo, compactação, escarificação, qualidade do solo.

\section{ABSTRACT}

The compaction and the quality of the soil are constantly questioned for the definition of the no-till soil

\begin{abstract}
management. This study was aimed at evaluating the spatial variability of the soil resistance to penetration, bulk density and soil organic carbon stratification ratio in layers on a clayey Typic Eutrothox, in contiguous areas of no-tillage with rotation of cultures and no-tillage with succession of cultures associated the chiselling. The study was set up from May to June of 2002, in Palotina, west region of the state of Parana, Brazil. The samples for determination the soil bulk density, soil resistance to penetration and soil organic carbon stratification ratio were obtained in 120 points in $2 \times 5 \mathrm{~m}$ grid, in the $0.40 \mathrm{~m}$ depth. Soil resistance to penetration measures were made three times, in a period of 10 days, initiate after having interrupted precipitation. The soil bulk density did not explain the spatial dependence of the compaction of the soil. The soil resistance to penetration and soil organic carbon stratification ratio presented range of $17 \mathrm{~m}$. The temporal variation of the spatial dependence of the soil resistance to penetration happens in the 0.05-0.20m layers. Similares spatial e dependence of the soil resistance to penetration and soil organic carbon stratification ratio reveals the importance of the quality of the soil in the spatial and temporary stabilization of the compaction of the soil expresses for the soil resistance to penetration in no-tillage systems.
\end{abstract}

Key words: geostatistical, no-tillage, soil bulk density, soil compaction, chiselling, soil quality.

\section{INTRODUÇÃO}

A resistência do solo à penetração $(\mathrm{RP})$ constitui uma das variáveis físicas consideradas na avaliação da qualidade do solo em plantio direto, envolvendo questionamentos quanto à compactação e a conveniência ou não do revolvimento mecânico

\footnotetext{
${ }^{1}$ Instituto Agronômico do Paraná (IAPAR). Estação Experimental de Paranavaí, CP 564, 87701-970, Paranavaí, PR, Brasil. Email: fidalski@iapar.br. Autor para correspondência.

${ }^{2}$ Universidade Estadual de Maringá (UEM). Departamento de Agronomia. Av. Colombo, 5790, 87020-900, Maringá, PR, Brasil. E-
} mail: catormena@uem.br, acagonca@wnet.com.br, rsojunior@uem.br. 
deste sistema de manejo para reduzí-la e propiciar menores restrições ao desenvolvimento radicular das plantas. Os estudos de variabilidade espacial da RP têm sido realizados em um único momento em áreas de sistema convencional (GREGO \& VIEIRA, 2005) ou no sistema plantio direto (ABREU et al., 2003; SILVAet al., 2004). Nesses estudos, os semivariogramas da RP apresentaram variações da continuidade espacial nos alcances e espessuras das camadas de solo.

Em áreas de plantio direto, nos solos argilosos do norte e oeste do Paraná, tem sido utilizado o revolvimento por meio da escarificação mecânica superficial antes da semeadura da safra de verão. A intervenção destas áreas de plantio direto é atribuída à menor eficiência do sistema radicular das culturas em sucessão. Por outro lado, a rotação de culturas planejada, com a inclusão de espécies como aveia e nabo forrageiro, cultivadas no inverno, pode ser indicada como alternativa para a manutenção da qualidade física do solo.

No plantio direto, a RP apresenta estreita relação com a densidade do solo (Ds). Espacialmente são encontradas duas situações envolvendo estas variáveis: a RP não depende da continuidade espacial da Ds (ABREU et al., 2003), ou a RP depende da continuidade espacial da Ds (SOUZA et al., 2001).

Os estudos de variabilidade espacial da RP não têm considerado a possível dependência desta variável com os teores de carbono orgânico do solo (CO). Recentemente, FRANZLUEBBERS (2002) sugeriu a taxa de estratificação de carbono orgânico do solo (QCO) como indicador de qualidade do solo em sistema de plantio direto, que consiste no quociente dos valores de CO da camada superficial e subsuperficial do solo.

Os objetivos deste estudo foram avaliar a variabilidade espacial da RP, Ds e QCO e temporal da RPem camadas de um Latossolo Vermelho eutroférrico, textura muito argilosa, em áreas contíguas de sistemas de manejo plantio direto.

\section{MATERIAL E MÉTODOS}

O estudo foi realizado no campo experimental da Cooperativa Agrícola Mista Vale do Piquiri (COOPERVALE), localizada no município de Palotina, PR ( $24^{\circ} 20^{\prime}$ S e 5350' W). O solo corresponde a um Latossolo Vermelho eutroférrico textura muito argilosa, relevo plano (EMBRAPA, 1999). O clima é subtropical (Cfa), segundo a classificação de Köppen, sem estação seca definida.

Duas unidades de observação contíguas foram selecionadas para o presente estudo, com dois sistemas de manejo desenvolvidos desde 1993. O primeiro sistema de manejo caracterizava-se pelo plantio direto (PD), com a rotação de culturas (milho- trigo-soja-aveia-soja-nabo forrageiro). O segundo sistema de manejo consistia na escarificação do sistema antes da semeadura de soja precoce na safra de verão, seguida da semeadura de milho de segunda safra (safrinha) ou trigo, denominado de plantio direto revolvido (PDR). As escarificações foram realizadas quando o solo apresentava umidade adequada para o manejo do solo, utilizando-se um escarificador de cinco hastes espaçadas de $0,25 \mathrm{~m}$, com profundidade efetiva de trabalho de $0,30 \mathrm{~m}$ de profundidade.

Em cada uma das unidades de observação (PD e PDR), foram referenciados no espaço 60 pontos (10 x 45m, respectivamente, nas direções X e Y, em seis linhas espaçadas de $2 \mathrm{~m}$ com 10 pontos de amostragem em cada linha, espaçados entre si $5 \mathrm{~m}$ ), correspondendo a $450 \mathrm{~m}^{2}$. As unidades de observação com PD e PDR estavam separadas por uma faixa de $3 \mathrm{~m}$ paralela à direção Y, resultando em 12 linhas paralelas e 120 pontos de amostragem.

Em cada um dos 120 pontos de amostragem foram obtidos os valores de RP, Ds e CO. A medida da RP foi iniciada depois de cessada a precipitação, realizada em 28/05/2002 $\left(E_{1}\right), 04 / 06 / 2002\left(E_{2}\right)$ e 07/06/ $2002\left(E_{3}\right)$, consistindo de uma repetição por ponto de amostragem obtida com um penetrômetro modelo Solotest S-310, com anel dinamométrico, em intervalos de $0,05 \mathrm{~m}$ até $0,40 \mathrm{~m}$ de profundidade, e realizada sobre os resíduos culturais de soja, antes da semeadura da cultura do trigo. Paralelamente, na $\mathrm{E}_{1}$, amostras de solo no centro da camada de $0-0,20 \mathrm{~m}$ foram coletadas com anéis volumétricos de $0,05 \mathrm{~m}$ de diâmetro e de altura, para a determinação da Ds, conforme GROSSMAN \& REINSCH (2002). Nesta mesma época, amostras deformadas de solo foram coletadas no centro das camadas de 0-0,05, 0,05-0,10 e 0,10-0,20m de profundidade, para a determinação dos teores de CO pelo método Walkley \& Black (EMBRAPA, 1997). O QCO foi calculado pelo quociente dos teores de $\mathrm{CO}$ da camada de 0-0,05m e 0,10-0,20m de profundidade.

Os dados experimentais de RP, Ds, CO e QCO das respectivas camadas e épocas de amostragem foram submetidos às análises estatísticas isoladamente para cada um dos sistemas de manejo (PD e PDR; 60 pontos) e conjuntamente (PD-PDR;120 pontos), seguindo as seguintes análises estatísticas: a - teste da distribuição da normalidade (Shapiro-Wilk) e análise exploratória dos dados originais $\left[\left(\mathrm{Z}\left(\mathrm{x}_{i}\right)\right]\right.$, utilizando-se o software estatístico SAS (SAS INSTITUTE, 2001); b - análise dos dados para identificação de pontos discrepantes (LIBARDI et al., 1996); c - verificação da dependência espacial expressa pelo semivariograma experimental, por meio do estimador clássico ou Matheron: $\gamma^{*}(\mathrm{~h})=\frac{1}{2 \mathrm{~N}(\mathrm{~h})} \sum_{i=1}^{\mathrm{N}(\mathrm{h})}\left[\mathrm{Z}\left(\mathrm{x}_{i}\right)-\mathrm{Z}\left(\mathrm{x}_{i}+\mathrm{h}\right)\right]^{2}$, obtendo-se a 
semivariância estimada $\gamma^{*}(\mathrm{~h}) ; \operatorname{com} \mathrm{Z}\left(\mathrm{x}_{i}\right) \mathrm{e} \mathrm{Z}\left(\mathrm{x}_{i}+\mathrm{h}\right)$, sendo os pares de valores medidos em locais afastados entre $\mathrm{x}_{i}$ da distância $\mathrm{h}$ e $\mathrm{N}(\mathrm{h})$, o número de pares de pontos separados por uma distância $\mathrm{h}$; $\mathrm{d}$ - aos semivariogramas experimentais, ajustou-se um modẹlo matemático esférico: $\gamma^{*}(\mathrm{~h})=\mathrm{C}_{\mathrm{o}}+\mathrm{C}_{1}\left[\frac{3}{2}\left(\frac{\mathrm{h}}{\mathrm{a}}\right)-\frac{1}{2}\left(\frac{\mathrm{h}}{\mathrm{a}}\right)^{3}\right]$, para $0<\mathrm{h}<\mathrm{a}$ e $\gamma^{*}(h)=C_{0}+C_{1}$ para $h \geq a$, em que $\mathrm{C}_{\mathrm{o}}$ (Nugget ou efeito pepita); a (Range ou alcance) e $\mathrm{C}_{0}+\mathrm{C}_{1}$ (Sill ou patamar); e - a obtenção e a avaliação da isotropia dos semivariogramas foram realizadas com o software Variowin (PANNATIER, 1996); f - validação cruzada dos semivariogramas por meio da krigagem ordinária para obter $Z^{*}\left(x_{i}\right)($ ISAAKS \& SRIVASTAVA, 1989) e estimar os desvios de cada variável $\left[\mathrm{Z}\left(\mathrm{x}_{i}\right)-\mathrm{Z} *\left(\mathrm{x}_{i}\right)\right]$, utilizando-se o software Geo-EAS (ENGLUND \& SPARKS, 1991); g - teste de normalidade Shapiro-Wilk (SAS INSTITUTE, 2001) dos desvios [Z( $\left.\left.\mathrm{x}_{\mathrm{i}}\right)-\mathrm{Z}^{*}\left(\mathrm{x}_{\mathrm{i}}\right)\right]$ obtidos da validação cruzada, critério utilizado para selecionar os semivariogramas experimentais e estimar o alcance da continuidade espacial das variáveis; $h$ escalonamento dos semivariogramas experimentais pela variância da amostra de dados $(n=120)$ (ISAAKS \& SRIVASTAVA, 1989); i - distribuição espacial dos valores da RP, mapas tridimensionais, obtidos por interpolação via krigagem ordinária, utilizando os modelos ajustados aos semivariogramas experimentais (GOLDEN SOFTWARE, 1997). Após uma análise exploratória dos mapas para visualizar a distribuição espacial dos valores de RP em cada uma das épocas e camadas de solo, comprovou-se a viabilidade de reeditá-los com os valores médios de RP das camadas de cada uma das épocas, utilizando-se o semivariograma escalonado comum às épocas e camadas de solo. Este procedimento permitiu a redução de seis para três mapas, corroborando a interpretação da dinâmica da variabilidade espacial e temporal da RP.

\section{RESULTADOS E DISCUSSÃO}

A análise dos dados da densidade do solo (Ds) no domínio dos sistemas de manejo PD, PDR ou PD-PDR indicou que esta variável estava distribuída de forma aleatória no espaço, sem dependência espacial nos domínios estudados (Tabela 1). Os dados originais $\left[\mathrm{Z}\left(\mathrm{x}_{i}\right)\right]$ dessa variável apresentaram distribuição normal (Shapiro-Wilk: $\mathrm{W}=0,98$ e $\mathrm{P}<\mathrm{W}=0,10$ ), sendo representada pelo valor médio de $1,18 \mathrm{Mg} \mathrm{m}^{-3}$ e intervalo de confiança de $95 \%$ para a Ds entre $1,17 \mathrm{e} 1,19 \mathrm{Mg} \mathrm{m}^{-3}$, significativa pela distribuição $\mathrm{t}(\mathrm{P}<0,05)$ no domínio estudado, apresentando coeficiente de variação de $4,71 \%$. A Ds não permitiu explicar a continuidade espacial da compactação do solo em acordo com ABREU et al. (2003).

Os semivariogramas dos sistemas de manejo contíguos PD-PDR indicaram a dependência espacial da RP nas camadas de 0,05 a 0,20m de profundidade (Tabela 1), divergindo das profundidades superficial $(<0,05 \mathrm{~m})$ e subsuperficial $(0,30 \mathrm{~m})$ obtidas por ABREU et al. (2003) em um Argissolo sob plantio direto. Na profundidade de 0,20 a $0,40 \mathrm{~m}$ de profundidade, não foi constatada dependência da RP em nenhuma das camadas do domínio estudado, semelhante aos resultados obtidos por GREGO \& VIEIRA (2005). O fato de a RP não ter apresentado continuidade espacial nas camadas de 0-0,05m e 0,20-0,30m, em PD, PDR e PD-PDR, evidencia que a continuidade espacial na camada de 0,05-0,20m não foi exclusivamente dependente da ação das hastes do escarificador utilizado a $0,30 \mathrm{~m}$ de profundidade em PDR, justificando a exploração da RP no domínio PD-PDR.

A partir da seleção dos seis semivariogramas pela validação cruzada, considerando a distribuição normal dos resíduos da RP (Tabela 1; P>0,01), escalonando-os pelas variâncias das amostras, obtevese um único semivariograma ajustado pelo modelo esférico comum às épocas e camadas de solo, com alcance da RP de $17 \mathrm{~m}$ (Figura 1). O escalonamento destes semivariogramas não alterou os padrões da estrutura espacial da RP (Tabela 1 e Figura 1), fato também constatado por MERCANTE et al. (2003).

Os mapas indicam que os valores da RP apresentaram amplitudes diferenciadas dentro e entre os sistemas de manejo avaliados, dependentes das camadas e das épocas de amostragem (Figuras 2a,b,c). $\mathrm{Na}_{1}$, a área do PDR apresentou valores de RP de 1,5MPa, enquanto o PD foi de 2,0MPa. $\mathrm{Na} \mathrm{E}_{2}$, pode-se verificar que partes da área do PDR apresentaram redução da RP para valores de 1,0-1,5MPa. Já na $E_{3}$, observa-se, no PDR, aumento dos valores de RP para 1,5-2,0MPa. O PD também apresentou elevação dos teores de RP na $\mathrm{E}_{3}$, comparada a $\mathrm{E}_{2}$, apresentando valores de RP de 2,5-3,0MPa. A amplitude espacial da RP em um intervalo de 10 dias se deve a redistribuição da água nos poros das camadas do solo, refletindo 
Tabela 1 - Valores médios, probabilidade $(p)$ das distribuições normais pelo teste Shapiro-Wilk (W) e coeficientes do modelo de semivariograma esférico para a densidade do solo (Ds), a resistência do solo à penetração (RP), o carbono orgânico (CO) e a taxa de estratificação de carbono orgânico do solo (QCO), por camada de solo e época de amostragem, comuns aos sistemas de manejo plantio direto com rotação de culturas (PD) e plantio direto com sucessão de culturas associado à escarificação a $0,30 \mathrm{~m}$ de profundidade (PDR).

\begin{tabular}{|c|c|c|c|c|c|c|c|c|c|c|c|c|}
\hline \multirow[t]{3}{*}{ Variável } & \multirow{3}{*}{$\begin{array}{c}\text { Camada } \\
\text { m }\end{array}$} & \multirow[t]{3}{*}{ Época } & \multicolumn{3}{|c|}{ Valores médios } & \multicolumn{4}{|c|}{ Teste Shapiro-Wilk (n=120) } & \multicolumn{3}{|c|}{ Semivariograma } \\
\hline & & & \multirow{2}{*}{$\begin{array}{l}P D \\
n=60\end{array}$} & \multirow{2}{*}{$\begin{array}{l}\text { PDR } \\
n=60\end{array}$} & \multirow{2}{*}{$\begin{array}{l}\text { PD-PDR } \\
n=120\end{array}$} & \multicolumn{2}{|c|}{$\mathrm{Z}\left(\mathrm{x}_{i}\right)$} & \multicolumn{2}{|c|}{$\mathrm{Z}\left(\mathrm{x}_{i}\right)-\mathrm{Z}^{*}\left(\mathrm{x}_{i}\right)$} & \multirow{2}{*}{\multicolumn{2}{|c|}{$\frac{\text { Pepita Patamar }}{\gamma(\mathrm{h}) \mathrm{s}^{-2}}$}} & \multirow{2}{*}{$\begin{array}{c}\text { Alcance } \\
\mathrm{m}\end{array}$} \\
\hline & & & & & & W & $p<\mathrm{W}$ & W & $p<\mathrm{W}$ & & & \\
\hline $\mathrm{Ds}^{(1)}$ & $0-0,20$ & $\mathrm{E}_{1}^{(2)}$ & 1,20 & 1,16 & 1,18 & 0,98 & 0,10 & - & - & - & - & - \\
\hline $\mathrm{RP}^{(1)}$ & $0-0,05$ & $\mathrm{E}_{1}$ & 4,11 & 1,38 & 2,75 & 0,94 & $<0,01$ & 0,96 & $<0,01$ & 0,45 & 3,27 & 17,05 \\
\hline $\mathrm{RP}$ & $0-0,05$ & $\mathrm{E}_{2}$ & 2,20 & 1,09 & 1,65 & 0,98 & 0,03 & 0,97 & $<0,01$ & 0,25 & 0,71 & 18,60 \\
\hline RP & $0-0,05$ & $\mathrm{E}_{3}$ & 2,94 & 1,82 & 2,38 & 0,97 & 0,02 & 0,96 & $<0,01$ & 0,17 & 0,86 & 24,18 \\
\hline $\mathrm{RP}$ & $\begin{array}{l}0,05- \\
0,10\end{array}$ & $\mathrm{E}_{1}$ & 3,59 & 2,12 & 2,86 & 0,99 & 0,22 & 0,95 & $<0,01$ & 0,66 & 1,50 & 18,60 \\
\hline $\mathrm{RP}$ & $\begin{array}{l}0,05- \\
0,10\end{array}$ & $\mathrm{E}_{2}$ & 2,32 & 1,48 & 1,90 & 0,99 & 0,72 & 0,98 & 0,14 & 0,10 & 0,38 & 20,46 \\
\hline $\mathrm{RP}$ & $\begin{array}{l}0,05- \\
0,10\end{array}$ & $\mathrm{E}_{3}$ & 3,04 & 2,17 & 2,61 & 0,99 & 0,39 & 0,98 & 0,18 & 0,25 & 0,67 & 26,35 \\
\hline $\mathrm{RP}$ & $\begin{array}{l}0,10- \\
0,15\end{array}$ & $\mathrm{E}_{1}$ & 2,83 & 1,99 & 2,41 & 0,96 & 0,68 & 0,99 & 0,29 & 0,28 & 0,54 & 18,60 \\
\hline $\mathrm{RP}$ & $\begin{array}{l}0,10- \\
0,15\end{array}$ & $\mathrm{E}_{2}$ & 2,23 & 1,58 & 1,91 & 0,99 & 0,77 & 0,99 & 0,26 & 0,18 & 0,33 & 21,08 \\
\hline $\mathrm{RP}$ & $\begin{array}{l}0,10- \\
0,15\end{array}$ & $\mathrm{E}_{3}$ & 2,89 & 2,32 & 2,60 & 0,91 & $<0,01$ & 0,91 & $<0,01$ & 0,05 & 0,07 & 21,70 \\
\hline $\mathrm{RP}$ & $\begin{array}{l}0,15- \\
0,20\end{array}$ & $E_{1}$ & 2,49 & 1,89 & 2,19 & 0,99 & 0,55 & 0,99 & 0,46 & 0,26 & 0,37 & 16,74 \\
\hline $\mathrm{RP}$ & $\begin{array}{l}0,15- \\
0,20\end{array}$ & $\mathrm{E}_{2}$ & 2,21 & 1,71 & 1,96 & 0,98 & 0,15 & 0,98 & 0,16 & 0,19 & 0,29 & 16,43 \\
\hline $\mathrm{RP}$ & $\begin{array}{l}0,15- \\
0,20\end{array}$ & $\mathrm{E}_{3}$ & 2,40 & 2,35 & 2,38 & 0,98 & 0,03 & - & - & - & - & - \\
\hline $\mathrm{CO}^{(1)}$ & $0-0,05$ & $\mathrm{E}_{1}$ & 19,03 & 18,91 & 18,97 & 0,99 & 0,28 & - & - & - & - & - \\
\hline $\mathrm{CO}$ & $\begin{array}{l}0,10- \\
0,20\end{array}$ & $\mathrm{E}_{1}$ & 12,27 & 15,21 & 13,74 & 0,99 & 0,46 & - & - & - & - & - \\
\hline $\mathrm{QCO}^{(3)}$ & & $\mathrm{E}_{1}$ & 1,73 & 1,28 & 1,51 & 0,98 & $0,12^{(4)}$ & 0,98 & 0,03 & 0,05 & 0,02 & 17,00 \\
\hline
\end{tabular}

${ }^{(1)} \mathrm{Ds}\left(\mathrm{Mg} \mathrm{m}^{-3}\right), \mathrm{RP}(\mathrm{MPa}), \mathrm{CO}\left(\mathrm{g} \mathrm{dm}^{-3}\right)$ e QCO (adimensional).

${ }^{(2)} \mathrm{E}_{1}-28 / 05 / 2002, \mathrm{E}_{2}-04 / 06 / 2002$ e $\mathrm{E}_{3}-07 / 06 / 2002$.

${ }^{(3)} \mathrm{QCO}=\mathrm{CO}_{0-10,05 \mathrm{~m}} / \mathrm{CO}_{0,10-0,20 \mathrm{~m}}$.

(4) Dados originais transformados para a obtenção da distribuição lognormal (ln).

nos teores de água de acordo com GREGO \& VIEIRA (2005).

A constatação da continuidade espacial de valores de $\mathrm{RP} \geq 2,0 \mathrm{MPa}$ em Latossolo Vermelho distroférrico típico sob PD, com alcance em torno de $17 \mathrm{~m}$ também foram observados por SILVA et al. (2004).
O fato de o revolvimento do PDR ter reduzido a $\mathrm{RP} \leq 2,0 \mathrm{MPa}$ não se deve à distribuição espacial da Ds, e tampouco do CO nas camadas de 0-0,05 e 0,10-0,20m (Tabela 1). Por sua vez, a estratificação de CO representado pelo QCO apresentou a mesma dependência espacial com alcance de $17 \mathrm{~m}$ (Tabela $1 \mathrm{e}$ 


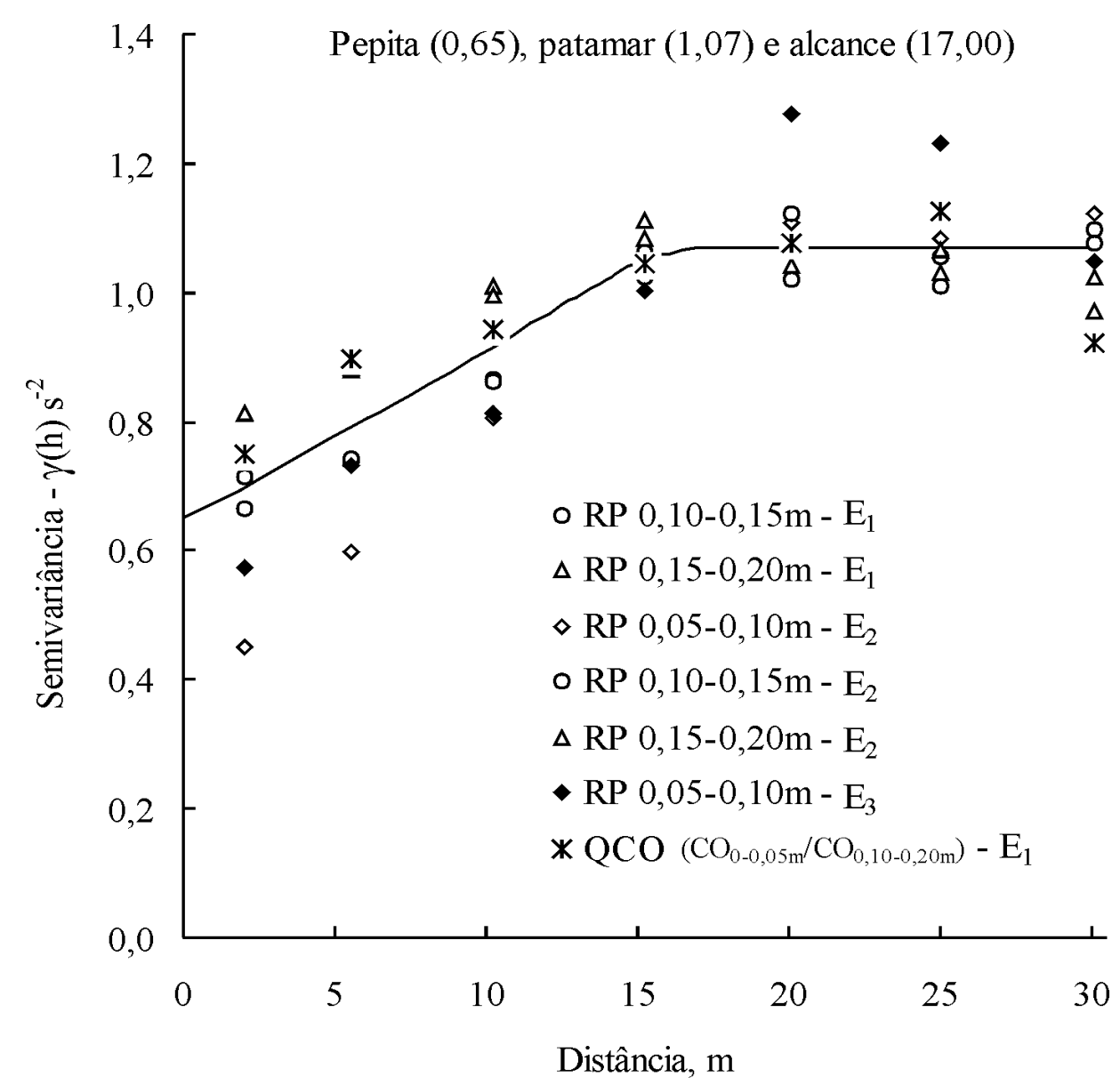

Figura 1 - Semivariograma escalonado da resistência do solo à penetração (RP) e taxa de estratificação de carbono orgânico do solo (QCO) em função das épocas de amostragem $\left(\mathrm{E}_{1}-28 / 05 / 2002, \mathrm{E}_{2}-04 / 06 / 2002\right.$ e $\left.\mathrm{E}_{3}-07 / 06 / 2002\right)$ e das camadas de solo, comuns aos sistemas de manejo plantio direto com rotação de culturas (PD) e plantio direto com sucessão de culturas associado à escarificação a $0,30 \mathrm{~m}$ de profundidade (PDR).

Figura 1). Os resultados obtidos por TORMENA et al. (2004) sugerem que a rotação de culturas em PD resultou em valores máximos de QCO de cerca de duas vezes aqueles obtidos no PDR, resultados semelhantes aos descritos por MRABET (2002) em plantio direto e convencional. Esta concentração superficial de CO é importante para a qualidade do solo no PD, uma vez que diferentes tipos de estresses relacionados com aplicação de agroquímicos, fertilizantes, precipitações e tráfego de máquinas incidem diretamente na superfície do solo (FRANZLUEBBERS, 2002). Neste contexto, o indicador de qualidade do solo QCO estabelece espacialmente as variações no sistema poroso do solo em relação aos efeitos da compactação do solo, que não foram expressos pela Ds e tampouco pelos teores de $\mathrm{CO}$.

\section{CONCLUSÕES}

A densidade do solo não explicou a dependência espacial da compactação do solo. A resistência do solo à penetração e a taxa de estratificação de carbono orgânico do solo apresentam alcances de $17 \mathrm{~m}$. A variação temporal da dependência espacial da resistência do solo à penetração ocorre nas camadas de 0,05-0,20m. As semelhanças da dependência espacial da resistência do solo à penetração e da taxa de estratificação de carbono orgânico do solo revelaram a importância da qualidade do solo na estabilização espacial e temporal da compactação do solo, expressa pela resistência do solo à penetração em sistemas de plantio direto.

Ciência Rural, v.36, n.6, nov-dez, 2006. 


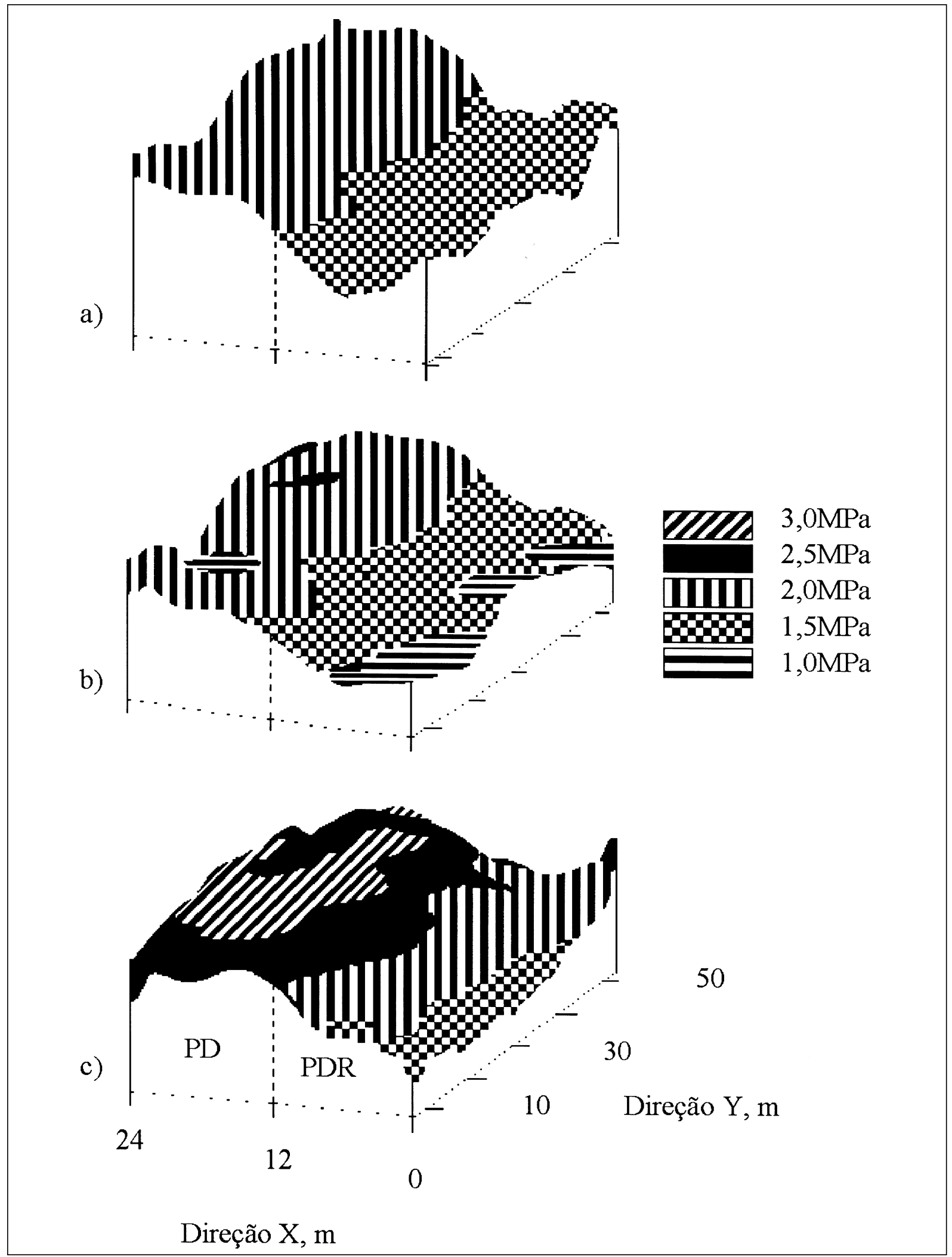

Figura 2 - Mapas da distribuição da resistência do solo à penetração (RP) para os sistemas de manejo plantio direto com rotação de culturas (PD) e plantio direto com sucessão de culturas associado à escarificação a $0,30 \mathrm{~m}$ de profundidade (PDR), na $\mathrm{E}_{1}-28 /$ 05/2002 a 0,10-0,20m (a), $\mathrm{E}_{2}-04 / 06 / 2002$ a $0,05-0,20 \mathrm{~m}$ (b) e $\mathrm{E}_{3}-07 / 06 / 2002$ a $0,05-0,10 \mathrm{~m}$ (c). 


\section{REFERÊNCIAS}

ABREU, S.L. et al. Variabilidade espacial de propriedades físicohídricas do solo, da produtividade e da qualidade de grãos de trigo em Argissolo franco arenoso sob plantio direto. Ciência Rural, v.33, p.275-282, 2003

EMBRAPA. Manual de métodos de análises de solos. Rio de Janeiro: Serviço Nacional de Levantamento e Conservação de Solos. 1997. 212p.

EMBRAPA. Sistema brasileiro de classificação de solos. Rio de Janeiro: Embrapa Solos, 1999. 412p. (EMBRAPA Produção de informação).

ENGLUND, E.; SPARKS, A. Geo-EAS - Geostatistical Enviromental Assessment Software. Las Vegas, U.S Enviromental Protency Agency, 1991. (EPA/600/4-88/033a).

FRANZLUEBBERS, A.J. Soil organic matter stratification ratio as an indicador of soil quality. Soil \& Tillage Research, v.66, p.95-106, 2002.

GOLDEN SOFTWARE. Surfer for windows - User's guide Release 6.0. USA, 1997. 340p.

GREGO, R.G.; VIEIRA, S.R. Variabilidade espacial de propriedades físicas do solo em uma parcela experimental. Revista Brasileira de Ciência do Solo, v.29, p.169-177, 2005 .

GROSSMAN, R.B.; REINSCH, T.G. Bulk density and linear extensibility. In: DANE, J.H.; TOPP, C. Methods of soil analysis, physical methods. Madison: Soil Science Society of America, 2002. v. 4, p.201-228.
ISAAKS, E.H.; SRIVASTAVA, R.M. An introdution to applied geostatistes. New York: Oxford University, 1989. 561p.

LIBARDI, P.L. et al. Variabilidade da umidade gravimétrica de um solo hidromórfico. Revista Brasileira de Ciência do Solo, v.20, p.1-12, 1996

MERCANTE, E. et al. Variabilidade espacial e temporal da resistência mecânica do solo à penetração em áreas com e sem manejo químico localizado. Revista Brasileira de Ciência do Solo, v.27, p.1149-1159, 2003.

MRABET, R. Stratification of soil aggregation and organic matter under conservation tillage systems in Africa. Soil \& Tillage Research, v,66, p.119-128, 2002.

PANNATIER, Y. Variowin: software for spatial data analysis in 2D. New York: Springer-Verlag, 1996. 90p.

SAS INSTITUTE. SAS/STAT User's guide. Version 8.2. Cary, NC, USA: Statistical Analysis System Institute, 2001. 943p.

SILVA, V.R. et al. Variabilidade espacial da resistência do solo à penetração em plantio direto. Ciência Rural, v.34, n.2, p.399406, 2004.

SOUZA, Z.M. et al. Variabilidade espacial de atributos físicos em um Latossolo Vermelho distrófico sob semeadura direta em Selvíria (MS). Revista Brasileira de Ciência do Solo, v.25, p.699-707, 2001.

TORMENA, C.A. et al. Propriedades físicas e taxa de estratificação de carbono orgânico num Latossolo Vermelho após dez anos sob dois sistemas de manejo. Revista Brasileira de Ciência do Solo, v.28, p.1023-1031, 2004. 